\title{
Experimental and Theoretical Study on the Symmetries of Orthogonally Polarized Optical Signals
}

\author{
William Shieh, Rongqing Hui, Xingwen Yi, and Graeme Pendock
}

\begin{abstract}
We perform theoretical analysis and systematic measurement of the degree-of-polarization and eye-closure penalty for optical signals with orthogonal polarizations. Both the theory and experiment show that the symmetry of the DOP is maintained for the orthogonal polarizations under both first and higher-order PMD, whereas the symmetry of eye-closure penalty is broken under second-order PMD. As a result, an orthogonal polarization pair can have large disparity of eye-closure penalty despite an identical degree-of-polarization. We also demonstrate a novel approach to estimate the maximum eye-closure penalty asymmetry with three orthogonal polarizations on the Poincare Sphere.
\end{abstract}

Index Terms-Optical communication, optical dispersion, optical fiber polarization, optical fibers, polarization mode dispersion.

\section{INTRODUCTION}

$\mathbf{P}$ OLARIZATION-MODE-DISPERSION (PMD) has become an inevitably active and practical topic as the optical transmission systems have advanced rapidly toward ultra-long reach of several thousand kilometers and ultra-high channel speed of $40 \mathrm{~Gb} / \mathrm{s}$ and beyond. The PMD distorts optical signal waveform and places a fundamental limit on the channel speed [1]-[2]. Additionally, under the influence of PMD, the optical signal will experience polarization dispersion and its degreeof-polarization (DOP) will degrade. Such DOP degradation has been proposed as an indicator to monitor fiber PMD [3][4]. On the other hand, when an optical signal traverses a PMD impaired medium, it has been rigorously proved that despite the effect of depolarization, the Stokes vectors of two input principal orthogonal polarizations will be an inversion of each other at the output, i.e., the directions of the Stokes vector are opposite while their magnitudes are equal [5]. In other words, the DOPs (the magnitude of the normalized Stokes vector) of the two principal orthogonal polarizations are symmetric

Paper approved by K. Kitaya, the Editor for Photonic Networks and Fiber Optic Wireless of the IEEE Communications Society. Manuscript received October 13, 2006; revised January 7, 2007. This work was supported by the Australian Research Council.

W. Shieh and G. Pendock are with the ARC Special Research Centre for Ultra-Broadband Information Networks, Department of Electrical and Electronic Engineering, The University of Melbourne, Melbourne, VIC 3010, Australia (e-mail: \{wshieh, gpendock\}@ee.unimelb.edu.au).

X. Yi is with National ICT Australia, Victory Research Laboratory, Department of Electrical and Electronic Engineering, The University of Melbourne, Melbourne, VIC 3010, Australia (e-mail: xyi@ee.unimelb.edu.au).

R. Hui is with the Electrical Engineering and Computer Science Department, the University of Kansas, Lawrence, KS 66045-7621 USA (e-mail: rhui@ku.edu).

Digital Object Identifier 10.1109/TCOMM.2009.0901.060464 for any-order of PMD. In this paper, we extend the theory to any arbitrary orthogonal polarization pair. Additionally, there is no systematic experimental verification for this symmetry theory in the open literature. Furthermore, it has been shown that the second-order PMD induced system impairment can be considered as the interference between the second-order PMD vector and fiber chromatic dispersion or phase chirp [6]. The same principle was successfully applied to the higherorder PMD compensation [7]. The existence of second-order PMD will consequently break the symmetry for the system penalty, i.e., the system penalties are no longer identical for two orthogonal polarizations, because the penalty is dependent on whether the input polarization is in- or out-of- phase with the second-order PMD vector [6]. In this paper, we first start with the theoretical derivation extending the DOP symmetry theory to any arbitrary polarization pairs. We then derive a close-form formula for system penalty asymmetry for an orthogonal polarization pair in the presence of secondorder PMD and chromatic dispersion. We then perform a systematic experimental study on the symmetry for orthogonal polarizations for both DOP and system penalty. We find that the symmetry of DOP is maintained for the orthogonal polarizations under both first and higher-order PMD, whereas the symmetry of eye-closure penalty is broken under secondorder PMD. We believe that this is the underlining justification for why DOP does not precisely predict the system impact of PMD when higher-order PMD is present. Optical signals with orthogonal polarizations would have the same DOP, but their system penalties may be quite different. In comparison, the RF power derived from the optical signal correlates very well with the system penalty, and is subsequently better suited as a monitor signal. We also demonstrate a novel approach to estimate the maximum eye-closure penalty asymmetry using three orthogonal polarizations on the Poincare Sphere.

\section{THEORETICAL BACKGROUND}

\section{A. Degree of Polarization}

A time dependent electric field $\psi_{o}(t)$ for a pulse at the output of the fiber can be generalized as a Jones vector:

$$
\psi_{o}(t)=\left(\begin{array}{l}
c(t) \\
d(t)
\end{array}\right)
$$

The associated Stokes vector of the optical signal, $\vec{S}$ can 
be formally expressed as

$$
\vec{S} \equiv \int_{-\infty}^{\infty} \vec{S}_{o}(t) \cdot d t
$$

where $\vec{S}_{o}(t)$ is the Stokes vector in time domain given by

$$
\vec{S}_{o}(t)=\psi_{o}^{+}(t) \cdot \vec{\sigma} \cdot \psi_{o}(t)
$$

where $\vec{\sigma}$ is the Pauli matrix vector [5], and the dagger denotes the Hermitian conjugate. It is also very useful to perform analysis in frequency domain through Fourier transform given by

$$
\Psi_{o}(\omega)=\frac{1}{\sqrt{2 \pi}} \int_{-\infty}^{\infty} \psi_{o}(t) \cdot e^{-i \omega t} \cdot d t
$$

where $\Psi_{o}(\omega)$ is the Fourier transform of the time domain signal $\psi_{o}(t)$, which is assumed to be normalized to 1 , i.e., $\int_{-\infty}^{\infty} \psi_{o}(t)^{+} \psi_{o}(t) d t=1$

Following Poole's framework in which the fiber is a linear medium without polarization dependent loss [1], we have:

$$
\begin{aligned}
\Psi_{o}(\omega) & =e^{j \phi_{D}(\omega)} \cdot U(\omega) \cdot \Psi_{i}(\omega) \cdot \varphi \\
& =T(\omega) \cdot \Psi_{i}(\omega) \cdot \varphi
\end{aligned}
$$

where we denote $T(\omega)=e^{j \phi_{D}(\omega)} \cdot U(\omega), \phi_{D}$ is real accounting for the chromatic dispersion effect, $U(\omega)$ is the unitary Jones matrix capturing the polarization dispersion effect in the fiber [1], $\Psi_{i}(\omega)$ (scalar) is the Fourier transform of the input electric field, and Jones vector $\varphi$ is the input state of polarization [8]. Combining Eqs. 2 to 5, we arrive at

$$
\vec{S}=\int_{-\infty}^{\infty} \vec{S}(\omega) \cdot\left|\Psi_{i}(\omega)\right|^{2} \cdot d \omega
$$

where $\vec{S}(\omega) \equiv \varphi^{+}(\omega) \cdot \vec{\sigma} \cdot \varphi(\omega)$, and $\varphi(\omega) \equiv U(\omega) \cdot \varphi$.

We will focus our analysis to the signals with the orthogonal polarizations denoted $\varphi_{+}$and $\varphi_{-}$, where subscript '+' and '-' stand for positive polarization and negative polarization. From the orthogonally of $\varphi_{+}$and $\varphi_{-}$, we further have

$$
\begin{aligned}
\varphi_{+}^{+}(\omega) \cdot \varphi_{-}(\omega) & =\varphi_{+}^{+} \cdot U^{+} U \cdot \varphi_{-} \\
& =\varphi_{+}^{+} \cdot \varphi_{-}=0
\end{aligned}
$$

or the output polarizations at each frequency $\omega, \varphi_{+}(\omega)$ and $\varphi_{-}(\omega)$ are also orthogonal. From Eq. 6, we can derive the following relationship for output Stokes Vectors at each frequency $\omega$ given by

$$
\vec{S}_{-}(\omega)=-\vec{S}_{+}(\omega)
$$

Eq. 8 is simply a widely recognized fact that the Stokes vectors for orthogonal polarizations are anti-parallel in Poincare Sphere. Substituting Eq. 8 into Eq. 6, we arrive at

$$
\vec{S}_{-}=-\vec{S}_{+}
$$

Eq. 9 shows that the Stokes vectors for the output signals from two orthogonal input polarizations are mirror image about the origin, i.e., they are equal in amplitude and opposite in direction. Using the known identity that

$$
D O P=|\vec{S}|
$$

we conclude that the degree-of-polarizations (DOP) for the output signals from two orthogonal polarizations are identical, or symmetric. This statement is true regardless of any form of the PMD in the transmission media.

\section{B. System Penalty}

We then study the system penalty for the optical signals with two orthogonal polarizations. It is a good approximation that the system penalty can be considered proportional to the pulse broadening. The pulse broadening, or the rms width $\delta$ of the any optical pulse can be defined as

$$
\delta^{2}=\left\langle t^{2}\right\rangle-\langle t\rangle^{2}
$$

where in the context of PMD, we find that [9],

$$
\begin{gathered}
\langle t\rangle=t_{0}-\frac{1}{2} \vec{\Omega}_{p} \cdot \vec{J} \\
\left\langle t^{2}\right\rangle=-\int_{-\infty}^{\infty}\left|\Psi_{i}\right|^{2}\left|\phi^{\prime} \cdot \vec{J}+\frac{1}{2} \vec{\Omega}\right| \\
+\left(\left|\Psi_{i}\right|^{\prime}\right)^{2} \cdot \frac{d \omega}{2 \pi} \\
\left|\Psi_{i}\right| \exp (i \phi)=\Psi_{i} e^{\left(i \phi_{D}\right)} \\
t_{0}=-\int_{-\infty}^{\infty}\left|\Psi_{i}\right|^{2} \phi^{\prime} \frac{d \omega}{2 \pi} \\
\vec{\Omega}_{p}=\int_{-\infty}^{\infty}\left|\Psi_{i}\right|^{2} \vec{\Omega} \cdot \frac{d \omega}{2 \pi}
\end{gathered}
$$

where $\Psi_{i}$ is the Fourier transform of electric field of the input optical pulse. $\phi_{D}$ is the phase from net fiber chromatic dispersion. $\phi$ is the phase of $\Psi_{i} e^{\left(i \phi_{D}\right)} \cdot \vec{J}$ is the input polarization as a Stokes vector. $\vec{\Omega}$ is the input PMD vector, with a magnitude of the DGD and a direction of the negative delay state. $t_{0}$ is the polarization independent delay. $\vec{\Omega}_{p}$ is the principal state of polarization (PSP) for the pulse, which may not be equal to the PSP at the center wavelength [5]. The prime indicates the derivative over angular frequency $\omega$. For the sake of the simplicity, we assume that only the linear phase chirp and the fiber chromatic dispersion are present on top of any arbitrary order of PMD. Expanding the phase $\phi$ at the center frequency $\omega_{0}$ and truncate at the second-order, we have

$$
\phi^{\prime \prime}=\phi_{0}^{\prime \prime}+\beta_{2}
$$

where $\phi_{0}^{\prime \prime}$ is the initial phase chirp and $\beta_{2}=\phi_{D}^{\prime \prime}$ is the fiber chromatic dispersion constant and equals to $\frac{-\lambda^{2}}{2 \pi c} D$, where $D$ is commonly used fiber chromatic dispersion [10], $\lambda$ is the center wavelength, $c$ is the speed of light. Combining Eqs.11-17, we 


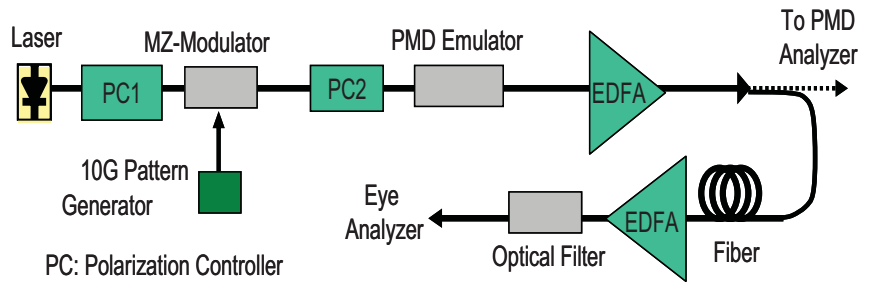

Fig. 1. Experimental setup for the symmetry study of orthogonal polarizations.

derive the pulse distortion difference between two orthogonal polarizations given by

$$
\left|\delta_{+}^{2}-\delta_{-}^{2}\right|=\left|\phi^{\prime \prime} \vec{J} \cdot \vec{\Omega}^{\prime}\right|=\left|\frac{\lambda^{2}}{2 \pi c} D_{e} \vec{J} \cdot \vec{\Omega}^{\prime}\right|(\Delta \omega)^{2}
$$

where $\delta_{+}^{2} / \delta^{2}$ is the pulse broadening for positive/negative polarization, $\vec{\Omega}^{\prime}$ is the second-order PMD vector, $(\Delta \omega)^{2}=$ $\left\langle\left(\omega-\omega_{0}\right)^{2}\right\rangle$ is the variance of the angular frequency $\omega$, and effective chromatic dispersion $D_{e} \equiv D-\frac{2 \pi c}{\lambda^{2}} \phi_{0}^{\prime \prime}$. For a Gaussian pulse with an RMS pulse width of $T_{0}$ and a chirp parameter of $\alpha, \phi_{0}^{\prime \prime}$ will be equal to $\frac{2 \alpha T_{0}^{2}}{1+\alpha^{2}}$.

Assuming the system penalty is proportional to the pulse broadening, from Eq.18, the system penalty asymmetry $\Delta P$ between the two polarizations are thus given by

$$
\Delta P=A\left|D_{e} \vec{J} \cdot \vec{\Omega}^{\prime}\right|
$$

where $A$ is a proportional constant.

There are two interesting results from Eq. 19. First, if only the first-order PMD is present $\left(\vec{\Omega}^{\prime}=0\right)$, the system penalty is identical or symmetric for two orthogonal polarizations $(\Delta P=0)$, and secondly, if second-order PMD is present and there is residual effective chromatic dispersion, the system penalty is asymmetric, or the system penalty symmetry is broken.

\section{EXPERIMENTAL SETUP}

We devise an experiment to verify the symmetry theory related to both DOP and system penalty. Fig. 1 shows the experimental set up for our symmetry study of DOP and eye-closure penalty for orthogonal polarizations. An intensitymodulated $10 \mathrm{~Gb} / \mathrm{s}$ PRBS optical signal is generated by an Agilent tunable laser and a JDSU Mach-Zehnder modulator. The optical signal passes through a PMD emulator, which consists either one-stage or two-stages of polarization-maintaining (PM) fiber to emulate first-order or higher-order PMD. The statistical distribution of the PMD is not a consideration for this paper. After traversing the PMD emulator, the signal is amplified before entering a fiber link. The output signal from the fiber link is further amplified by a second EDFA, optically filtered and fed into an Agilent high-speed oscilloscope equipped with a wideband optical detector. The optical filter $3 \mathrm{~dB}$ bandwidth is $1 \mathrm{~nm}$ and the OSNR of the optical signal is approximately $25 \mathrm{~dB}$ measured with a resolution bandwidth of $0.1 \mathrm{~nm}$. Eye-closure penalty has been used to characterize the PMD-induced system impairment [11] and is adopted in this paper. For any practical purpose, Q parameter penalty can be considered to be proportional to eye-closure penalty. Subsequently, the measured relationship between eye-closure penalty and DOP can be considered to be that between $\mathrm{Q}$ penalty and DOP. The signal waveform traces are averaged to remove the random noise and recorded. Then the waveform data is converted into eye-diagrams through a Matlab program to compute the eye-closure penalty due to PMD-induced waveform distortion. The signal Stokes vector is measured using an Agilent polarization analyzer by tapping the optical signal before it enters the fiber link. The measured Stokes vectors are related to those launched into the fiber by a simple Stokes vector rotation. Because the fiber link has negligible PMD, the DOP symmetry is not altered by further passing through the fiber link. The rationale to monitor the Stokes vector before the fiber link is that in order to measure the symmetry/asymmetry of orthogonal polarization pairs as a function of the fiber chromatic dispersion, several different spools of transmission fibers are used for the fiber link during the measurement. By placing the polarization analyzer before the transmission fiber, the stability of the PMD emulator is monitored. The first polarization controller PC1 is used to optimize the input polarization for the MZ-modulator, and the second polarization controller PC2 is used to systematically adjust the launch polarization into the PMD emulator for $\mathrm{PMD} /$ Stokes vector analysis. There is an additional PC (not shown in Fig. 1) before PC2 to ensure that the signal enters the half-wave plate (HWP) in PC2 with a linear polarization. The systematic polarization adjustment is done by physically rotating the HWP in $\mathrm{PC} 2$ for $90^{\circ}$, which corresponds to the Stokes vector rotating of 360 degree along a maximum circle on Poincare Sphere.

Both the DOP and the eye-closure penalty are functions of launch polarization (or input Stokes vector) into PMD emulator. Although the results can be presented by a threedimensional polar plot, it would be quite complex and the intuitive picture might be lost. Instead, we show a twodimensional polar plot presenting the DOP or eye-closure penalty as a function of the input polarization. When the launch polarization is rotated from 0 to $360^{\circ}$ on the Poincare Sphere, the corresponding DOPs are measured with the polarization analyzer, and the signal waveform traces detected at the end of the transmission fiber spool are recorded, from which the eye-closure penalty is computed.

\section{Measurement and Discussion}

The symmetry measurement for the eye-closure penalty with the transmission fiber chromatic dispersion of $0 \mathrm{ps} / \mathrm{nm}$ and $-1360 \mathrm{ps} / \mathrm{nm}$ are shown in Figs. 2(a) and (b) respectively. The negative dispersion of $-1360 \mathrm{ps} / \mathrm{nm}$ is obtained with a dispersion compensation module (DCM-80) which is designed to compensate the dispersion of $80 \mathrm{~km}$ standard single mode fiber (SSMF). In this measurement, only one stage of PM fiber is used in the PMD emulator with 50ps DGD and therefore only first-order PMD is involved. In Fig.2 (a), $0 \mathrm{~km}$ transmission fiber is used and each pair of orthogonal polarizations is represented by two data points which are $180^{\circ}$ apart on the two-dimensional polar plot. For instance, the measurements made at $80^{\circ}$ and $260^{\circ}$ is one of the orthogonal polarization pairs, represented by A and B in Fig. 2(a). We 


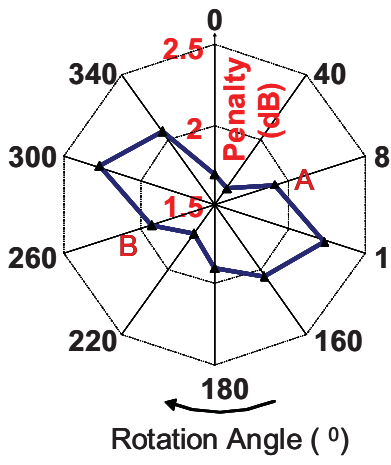

(a)

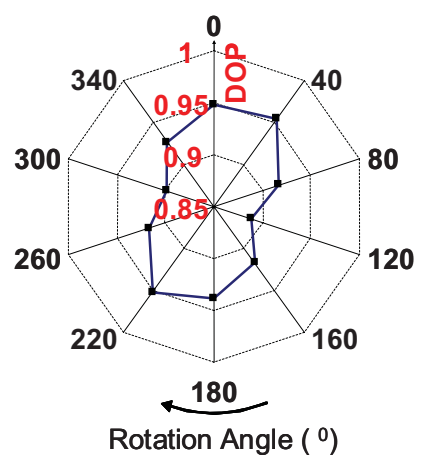

Fig. 2. Polar plots for (a) the eye-closure penalty for $0 \mathrm{~km}$ fiber, (b) the eyeclosure penalty for $80 \mathrm{~km}$ dispersion-compensation-module (DCM) fiber, and (c) the DOP for $0 \mathrm{~km}$ fiber. The polar angle represents the angle of launched signal polarization state and the radius indicates the eye-closure penalty or DOP. The tests are done with a single-stage $50 \mathrm{ps}$ PM fiber. The dashed lines in the figure are the equal value contours for eye-closure penalty or DOP. Points A and B in Fig. 2 (a) is one example of a pair of orthogonal polarizations.

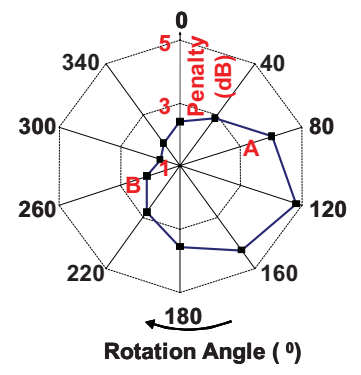

(a)

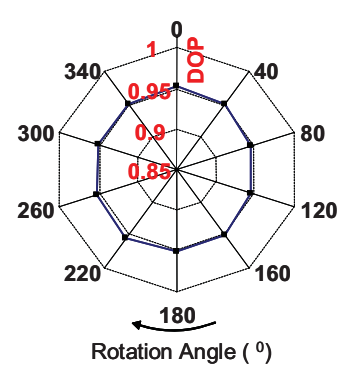

(b)
Fig. 3. The polar plot for (a) eye-closure penalty for $80 \mathrm{~km}$ dispersioncompensation-module DCM fiber, and (b) DOP. The tests are done with twostage PM fiber as a PMD emulator.

can see that all the orthogonal polarization pairs result in the same penalty, as evidenced by the symmetry of the data points for orthogonal pairs, for instance, data point A is almost an inversion of B across the origin. Such symmetry remains intact when $80 \mathrm{~km}$ of DCM fiber is introduced as shown in Fig. 2(b). We also measure the symmetry of eye-closure penalty with $26 \mathrm{~km}, 50 \mathrm{~km}$ and $76 \mathrm{~km} \mathrm{SSMF}$, corresponding to the chromatic dispersion of $442 \mathrm{ps} / \mathrm{nm}, 850 \mathrm{ps} / \mathrm{nm}$, and 1292 $\mathrm{ps} / \mathrm{nm}$, respectively. The penalty symmetry remains intact for

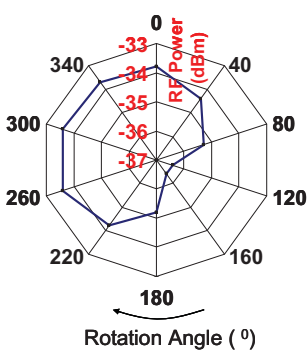

(a)

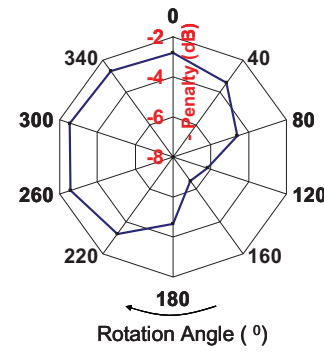

(b)
Fig. 4. Polar plots for (a) the RF spectral density at $5 \mathrm{GHz}$ for $80 \mathrm{~km}$ dispersion-compensation-module (DCM), and (b) the eye-closure penalty for $80 \mathrm{~km}$ dispersion-compensation-module (DCM) fiber. The tests are done with two-stage 50 ps PM fiber as a PMD emulator.

varying chromatic dispersion values we use. Similarly Fig. 2(c) shows the measured DOP on the polar plot, and clearly each orthogonal polarization pair has the same DOP.

Fig. 3(a) shows the symmetry study for eye-closure penalty when the optical signal passes through a two-stage (50 ps +50 ps DGD) PMD emulator with the eigen-axes of the two stages misaligned with each other so as to generate higher-order PMD. The first-order PMD and second PMD are measured to be 33 ps and $1500 \mathrm{ps}^{2}$ respectively in this experiment. The asymmetry of the eye-closure penalty is proportional to the magnitude of the effective chromatic dispersion and secondorder PMD [Eq.19], therefore only the result obtained with large chromatic dispersion of $-1360 \mathrm{ps} / \mathrm{nm}$ from $80 \mathrm{~km}$ DCM is shown in Fig. 3(a). We see that even though the DOP is still symmetric for each orthogonal polarization pair as shown in Fig. 3(b), the symmetry of the eye-closure penalty is broken. For example, the penalties for the orthogonal pair at the angles of $120^{\circ}$ and $300^{\circ}$ are $1.7 \mathrm{~dB}$ and $4.8 \mathrm{~dB}$ respectively. This represents a $3 \mathrm{~dB}$ asymmetry despite an identical DOP of $95 \%$ at these two launch polarizations. This further illustrates the inaccuracy of DOP as a PMD degradation monitor. This signifies that DOP may be a good indicator for the firstorder PMD, but does not predict the second-order PMD, and subsequently is not a good feedback signal for a system with large higher-order PMD [12].

The RF power from the detected signal has also been used as a feedback signal for PMD compensation [13]. We conduct an experiment to verify the correlation between RF signal power and eye closure penalty. The optical signal at the output of the fiber spool is detected with wideband optical detector, and the RF signal from the detector is fed into a RF spectrum analyzer. The RF spectral density is measured at $5 \mathrm{GHz}$. Due to the polarization drift in the setup, the launched polarizations are different than those used in Fig. 3. Fig. 4 shows the measured RF spectral density at $5 \mathrm{GHz}$ and corresponding eye-closure penalty. The eye-closure penalty is plotted with a negative value in order to facilitate the comparison. As we can see that RF spectral density at $5 \mathrm{GHz}$ correlates very well with the eye-closure penalty. This is evidenced by the similar asymmetry of RF signal to that of eye-closure penalty, in terms of their overall shape and where the maximum asymmetry takes place. The corresponding maximum asymmetries are 3.9 $\mathrm{dB}$ and $2.8 \mathrm{~dB}$ for eye-closure penalty and RF spectral density, respectively, which takes place at the polarization pair of $120^{\circ}$ 
and $300^{\circ}$.

We next quantitatively investigate the eye-closure penalty asymmetry for different fiber chromatic dispersions when the two-stage (50ps+50ps DGD) PMDE are still used. From Eq. 19 , the maximum asymmetry $\Delta P_{\max }$ occurs when the two launch polarizations are in- or out-of- phase with the secondorder PMD vector, given by

$$
\Delta P_{\max }=A \cdot\left|D-\frac{2 \pi c}{\lambda^{2}} \phi_{0}^{\prime \prime}\right| \cdot\left|\vec{\Omega}^{\prime}\right|
$$

In general, the maximum asymmetry may not be reached for orthogonal polarizations, but it can be measured by launched three orthogonal pairs, for instance, (i) $(1,0,0)$ and $(-1,0,0)$, (ii) $(0,1,0)$ and $(0,-1,0)$, and (iii) $(0,0,1)$ and $(0,0,-1)$ on the Poincaré Sphere. It is easy to show that from Eqs. 19 and 20 that

$$
\Delta P_{\max }=\sqrt{\left(\Delta P_{1}\right)^{2}+\left(\Delta P_{2}\right)^{2}+\left(\Delta P_{3}\right)^{2}}
$$

where $\Delta P_{1-3}$ is the asymmetry of eye-closure penalty associated with each of the three orthogonal pairs on the Poincare Sphere. From Eq. 21, the maximum penalty asymmetry can be computed with the penalty values at these three pairs of orthogonal polarizations. Additionally, the maximum eyeclosure penalty asymmetry is a linear function of the chromatic dispersion with the slope proportional to the second-order PMD [Eq. 20]. In our experiment, we measure the eye-closure penalties for the three orthogonal pairs, and use Eq. 21 to derive maximum eye-closure penalty asymmetry. Fig. 5 shows the maximum penalty asymmetry at chromatic dispersions of $0 \mathrm{ps} / \mathrm{nm}, 442 \mathrm{ps} / \mathrm{nm}$, and $850 \mathrm{ps} / \mathrm{nm}$. The solid line is the straight-line fit for the experimental data. Interestingly, the minimum penalty asymmetry occurs at $-195 \mathrm{ps} / \mathrm{nm}$ due to the chirp induced by the optical modulator, which is independently verified. We use the same coefficients to predict the eyeclosure asymmetry for the chromatic dispersion of -1360 $\mathrm{ps} / \mathrm{nm}$ from $80 \mathrm{~km}$ of DCM fiber. The measured data shows 4.2 $\mathrm{dB}$ asymmetry compared with the $3.5 \mathrm{~dB}$ asymmetry predicted by theory. The difference is attributed to the relatively large eye-closure penalty around $6 \mathrm{~dB}$ for the $80 \mathrm{~km}$ DCM fiber along with the impact of second-order PMD. The DOPs of three orthogonal pairs for each chromatic dispersion of transmission fiber are also recorded, showing intact symmetry in spite of corresponding large eye-closure asymmetry. It can be shown that the mean value of the second-order PMD for a fiber link of a mean PMD of $50 \mathrm{ps}$ is about $1400 \mathrm{ps}^{2}$. Therefore the level of the penalty asymmetry we have observed in Fig. 5 corresponds approximately to that of a fiber link with a mean PMD of 50 ps.

\section{CONCLUSION}

We have performed theoretical analysis and systematic measurement of the degree-of-polarization and eye-closure penalty for optical signals with orthogonal polarizations. Both the theory and experiment show that the symmetry of the DOP is maintained for the orthogonal polarizations under either first or higher-order PMD, whereas the symmetry in eye-closure penalty is broken under second-order PMD. As a result, an orthogonal polarization pair can have large disparity in eyeclosure penalty despite an identical degree-of-polarization. We

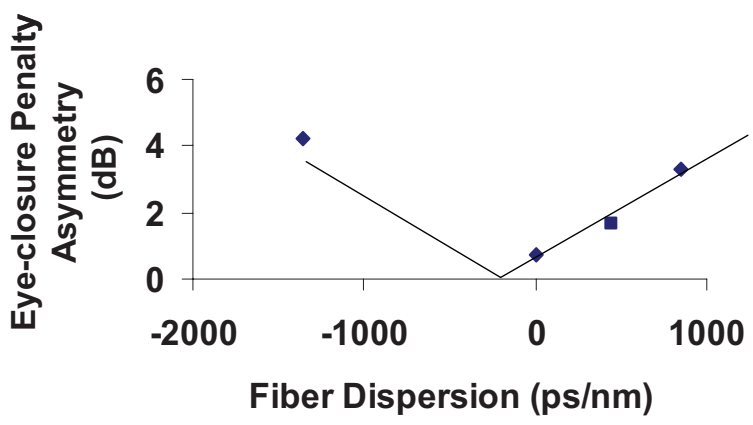

Fig. 5. Maximum eye-closure penalty asymmetry as a function of fiber dispersion.

also demonstrate a novel approach to estimate the maximum eye-closure penalty asymmetry with three orthogonal polarizations on the Poincare Sphere.

\section{ACKNOWLEDGEMENT}

We would like to thank K. E. Cornick at the University of Melbourne for her insightful discussion and valuable laboratory assistance. This work was supported by the Australian Research Council (ARC). R. Hui was partially supported by US National Science Foundation award \# 0435381.

\section{REFERENCES}

[1] C. D. Poole and R. E. Wagner, "Phenomenological approach to polarization dispersion in long single-mode fibers," IEEE Electron. Lett., vol. 22, pp. 1029-1030, 1986.

[2] C. Poole, R. W. Tkach, A. R. Chraplyvy, and D. A. Fishman, "Fading in lightwave systems due to polarization-mode dispersion," IEEE Photon Technol. Lett., vol. 3, pp. 68-70, 1991.

[3] N. Kikuchi, "Analysis of signal degree of polarization degradation used as control signal for optical polarization mode dispersion compensation," J. Lightwave Technol., vol. 19, pp. 480-486, 2001.

[4] Y. W. Song, S. M. R. Motaghian Nezam, Z. Pan, and A. E. Willner, "Accurate DOP monitoring of several WDM channels for simultaneous PMD compensation," in Proc. Conf. Lasers Electro-Optics, May 2004, vol. 2, pp. 16-21.

[5] W. Shieh, "Principal states of polarization for an optical pulse," IEEE Photon Technol. Lett., vol. 11, pp. 677-679, 1999.

[6] W. Shieh, "On the second-order approximation of PMD," IEEE Photon Technol. Lett., vol. 12, pp. 290-292, 2000.

[7] L.-S. Yan, Q. Yu, T. Luo, A. E. Willner, and X. S. Yao, "Compensation of higher-order PMD using phase modulation and polarization control in the transmitter," IEEE Photon Technol. Lett., vol. 14, pp. 858-860, 2002.

[8] C. D. Poole and C. R. Giles, "Polarization-dependent pulse compression and broadening due to polarization dispersion in dispersion-shifted fiber," Optics Lett., vol. 13, pp. 155-157, 1988.

[9] M. Karlsson, "Polarization mode dispersion-induced pulse broadening in optical fibers," Optics Lett., vol. 23, pp. 688-690, 1998.

[10] G. P. Agrawal, Fiber-Optic Communication Systems. New York: John Wiley and Sons, 2002.

[11] J. H. Winters and M. A. Santoro, "Experimental equalization of polarization dispersion," IEEE Photon Technol. Lett., vol. 2, pp. 591-593, 1990.

[12] H. Miao and C. Yang, "Analysis of signal degree of polarization degradation induced by polarization-mode dispersion in optical fibers," IEEE Photon Technol. Lett., vol. 16, p. 2475-2477, 2004.

[13] H. Heismann, D. Fishman, and D. Wilson, "Automatic compensation of first-order polarization mode dispersion in a $10-\mathrm{Gb} / \mathrm{s}$ transmission system," in Proc. ECOC'98, vol. WDC11, pp. 529-530. 
William Shieh received his M.S. in Electrical Engineering-Communications and Ph.D. in physics both from the University of Southern California in 1994 and 1996, respectively.

From 1996 to 1998 , he worked as a member of technical staff for the Jet Propulsion Labs, Pasadena, California. From 1998 to 2000, he worked as a member of technical staff for Bell Labs, Lucent Technologies, Holmdel, New Jersey. From 2000 to 2003, he worked as a technical manager for Dorsal Networks, Columbia, Maryland. Since 2004, he has been with the department of Electrical and Electronic Engineering at the University of Melbourne as a senior lecturer. He has a broad interest in the field of optical communications including polarization effects in fibers, coherent communications systems, and optical packet switching. His current research interests include OFDM techniques in both wireless and optical communications, coherent optical communication systems, and optical packet switching. He has published more than 100 journal and conference papers, and submitted 14 U.S. patents (nine issued) covering areas of polarization controller, wavelength stabilization in WDM systems, and Raman amplifier-based systems and subsystems.

Rongqing Hui received a B.S. degree in Microwave Communications in 1989 and an M.S. degree in Lightwave Technology in 1988, both from Beijing University of Posts \& Telecommunications, Beijing, China. He received a $\mathrm{Ph} . \mathrm{D}$ degree in Electrical Engineering from Politecnico di Torino, Torino, Italy in 1993.

From 1982 to 1985, he taught at the Physics Department of Anhui University, Hefei, China, where he also conducted research on optical fibers and fiber sensors. From 1985 to 1989 , he was with the Optical Communication Laboratory of Beijing University of Posts \& Telecommunications, where he worked in coherent optical fiber communication systems and components. From 1989 to 1990, he held a research fellowship from the Fundazione Ugo Bordoni, Rome Italy, working on nonlinear effects and optical injection locking of semiconductor laser devices. From 1990 to 1993, he was with the Department of Electronics, Politecnico di Torino, where he worked on optical communications and single frequency semiconductor laser devices. During this period, he also worked as a research engineer at the Telecommunication Research Center (CSELT), Torino, Italy. He spent one year, from 1993 to 1994, as a postdoctoral research fellow working on optical systems and networks architecture at the University of Ottawa, Ont., Canada. He joined Bell-Northern Research (now part of Nortelnetworks), Ottawa, Canada in 1994 as a Member of Scientific Staff, where he has worked in the research and development of high-speed optical transport networks. Since September 1997, he has been with the department of Electrical Engineering and Computer Science at the University of Kansas, where he is a professor.
As an author or co-author, he has published more than 70 refereed technical papers in the area of fiber-optic communications and holds 11 patents. Dr. Hui currently serves as an Associate Editor of the IEEE JOURNAL OF QUANTUM ELECTRONICS.

Xingwen Yi received the B.Eng. degree in electronic engineering from Southeast University, Nanjing, China, in 1999 and the Ph.D. degree in electrical and electronic engineering from the University of Melbourne, Melbourne, Australia, in 2007.

From 1999 to 2004, he was at Huawei Technologies, Co., Ltd., China. He was involved in EDFA development, system design, and key technologies investigations for DWDM long-haul transmission systems. In 2005, he was an Intern for three months at Alcatel SEL AG, Germany, where he developed algorithms for feedforward equalizers and decision feedback equalizers. He is currently a Research Scientist at the University of California, Davis. He has published more than 30 journal and conference papers. He also holds seven patents in China. His current research interests include electronic compensation of optical distortions, all-optical label swapping techniques, and optical performance monitoring.

Graeme J. Pendock has B.Sc. (1988) and M.Sc (1990) degrees in electrical engineering from the University of Cape Town, South Africa, and a Ph.D. (1993) in engineering from the University of Cambridge, UK.

After his $\mathrm{PhD}$ he worked for Plessey-Tellumat (Cape Town) where he was involved in the development and manufacture of microwave products. In 1993 he joined The University of Melbourne as a Post-Doc where he worked on optical coherence multiplexed systems, spectral sliced transmission, ASE noise in systems, wavelength conversion, and optical transmission. He developed the optical transmission simulator that was later commercialized by Virtual Photonics. In 1998 he joined Bell Labs, Lucent, and then later worked for Sycamore Networks, MA. He is currently an Associate Professor in the Department of Electrical and Electronic Engineering at The University of Melbourne. 